\title{
Entwicklung neuartiger hierarchisch strukturierter Silika- Membranen für Anwendungen in der Biosensorik
}

\author{
Nicole Anders, Dirk Enke \\ Universität Leipzig, Institut für Technische Chemie; Linnéstraße 3, 04103 Leipzig
}

\section{Zusammenfassung}

Durch die Kombination von spinodaler Entmischung und Sol-Gel-Übergang wird eine bimodale Porenstruktur erzeugt. Dieses schwammartige Netzwerk wird dabei aus definierten Mikro- und Makroporen geformt. Durch eine hydrothermale Nachbehandlung mit Ammoniak-Lösung bei $120^{\circ} \mathrm{C}$ werden die Mikroporen in Mesoporen umgewandelt. Es ist möglich Mesoporen in einem Bereich von 6-60nm mit nahezu konstanter Makroporengröße zu generieren [1]. Membranen mit dieser bimodalen Porenstruktur (Bild 1) werden für Sensoranwendungen getestet. Um dafür ein Enzym kovalent an die Membran anzubinden, wird die Oberfläche mit einem Aminosilan funktionalisiert. Acetylcholinesterase wird als Enzym für den Trägermaterialtest verwendet. Im Vergleich mit einer CPGMembran (Bild 4) wird eine schnellere Ansprechzeit erreicht und die Inhibierung des Enzym-Sol-GelKomplexes für das Testpestizid Carbofuran konnte mit einer Nachweisgrenze von 0.125 $\mu \mathrm{g} / \mathrm{l}$ gezeigt werden. Für den Nachweis der Langzeitstabilität werden die immobilisierten Membranen bei $4^{\circ} \mathrm{C}$ in einer Pufferatmosphäre mit einem pH-Wert von 7 gelagert. Die Membranen zeigen nach 7 Wochen noch nahezu die Anfangsaktivität.

\section{Einleitung}

Enzymreaktionen, wie die katalytische Zersetzung des Substrates Acetylcholinchlorid durch das Enzym Acetylcholinesterase, werden oft für den Nachweis von Pestiziden eingesetzt [2,3]. Zumeist werden die Enzyme für solch eine Anwendung auf einem Träger fixiert. Trägermaterialien nach dem Sol-Gel-Prozess hergestellt, können je nach Herstellungsbedingungen in ihrer Porenstruktur eine Hierarchie aufweisen (Bild 1).

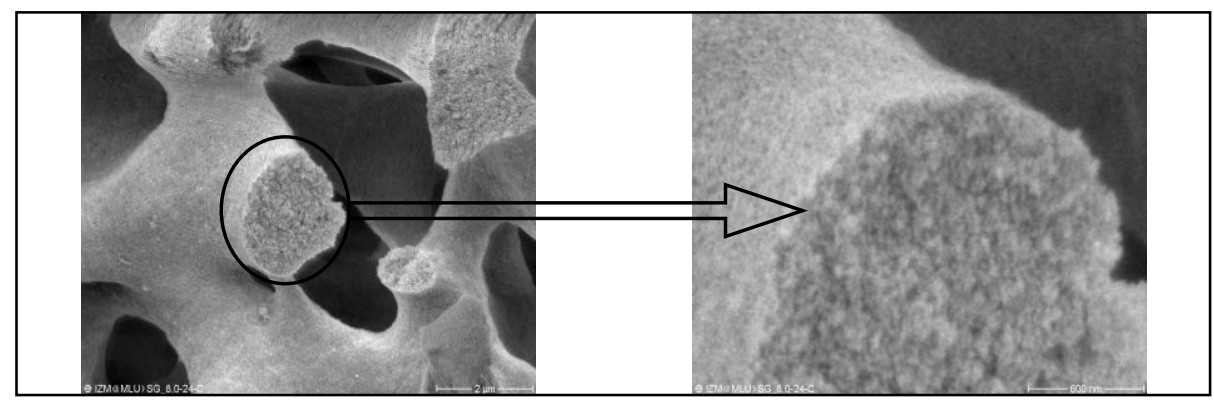

Bild 1 Elektronenmikroskopische Aufnahme des hierarchischen Sol-Gel-Netzwerkes

Das schwammartige Silikat-Netzwerk wird dabei aus definierten Mikro- und Makroporen geformt, welche als Reaktions- bzw. als Transportporen fungieren und somit in der Sensoranwendung schnellere Ansprechzeiten erzielen können. Bei der Fixierung der Aktivkomponente auf der Oberfläche ist zu beachten, dass das Enzym sowohl leaching stabil als auch ohne großen Aktivitätsverlust aufgebracht wird. Die kovalente Immobilisierung des Enzyms auf die spezifische Oberfläche des Sol-Gel-Materials mittels eines Spacers ist die am häufigsten verwendete Methode. Da die Mikroporen in den Netzwerkwänden für eine Enzymimmobilisierung einen zu geringen Porendurchmesser aufweisen, sind nicht alle Poren und somit nicht die gesamte Oberfläche des SolGel-Materials für die Funktionalisierung zugänglich. Um eine ausreichende Enzymkonzentration und 
schnelle Ansprechzeiten zu gewährleisten, werden die Mikroporen durch eine hydrothermale Reaktion vergrößert.

\section{Durchführung}

\subsection{Herstellung des Trägermaterials}

Bei der Herstellung der Sol-Gel-Membranen wird durch Kombination einer polymerinduzierten spinodalen Entmischung und eines Sol-Gel-Übergangs eine bimodale Porenstruktur aus Mikro- und Makroporen erzeugt. Die Zusammensetzung des Ausgangsmaterials ist in Tafel 1 dargestellt.

Tafel 1 Zusammensetzung des Ausgangsmaterials

\begin{tabular}{l|l|l}
\hline & Bezeichnung & Anteil \\
\hline Lösungsmittel & Wasser & $1 \mathrm{~mol}$ \\
\hline Säure & Schwefelsäure (konzentriert) & $25 \mathrm{mmol}$ \\
\hline Templat & Polyethylenoxid (100.000) & $0,025 \mathrm{mmol}$ \\
\hline Prekursor & Tetraethylorthosilikat & $70 \mathrm{mmol}$ \\
\hline
\end{tabular}

Um stabile Monolithe zu erzeugen, wird die von Preising [4] entwickelte Druck-LösungsmittelAustausch-Synthese durchgeführt. Hierbei erfolgt die Gelierung bei $50^{\circ} \mathrm{C}$ für 24 Stunden und einem Druck von 15 bar zur Verfestigung des Silikat-Netzwerkes. Danach wird ein Lösungsmittelaustausch von der Mutterlösung zu Ethanol und anschließend zu n-Pentan vorgenommen, um eine rissfreie Trocknung und somit eine höhere Stabilität des Materials zu gewährleisten. Dabei werden die Membranen unter Rückfluss in dem jeweiligen Lösungsmittel für 24 Stunden gekocht. Die Mikroporen werden durch eine hydrothermale Nachbehandlung [1] mit Ammoniumhydroxid-Lösung ( $1 \mathrm{~mol} / \mathrm{l}$ ) bei $120^{\circ} \mathrm{C}$ für 48 Stunden in Mesoporen umgewandelt (Bild 2). Die Membranen werden anschließend mit Wasser gewaschen, getrocknet und für 4 Stunden bei $500^{\circ} \mathrm{C}$ kalziniert.

\subsection{Enzymimmobilisierung auf die Trägeroberfläche}

Das Enzym Acetylcholinesterase (350U/mg Protein ee) wird kovalent an die Membranoberfläche angebunden (Bild 2). Hierzu wird die poröse Oberfläche im ersten Schritt mit dem Silanisierungsreagenz $\gamma$-Aminopropyltriethoxysilan (1 Ma.\%) funktionalisiert. Das Feststoff zu Lösungs-Verhältnis $(\mathrm{mg} / \mathrm{ml})$ beträgt 50:1. Die Reaktion verläuft zuerst eine Stunde bei RT und 600mbar und anschließend für zwei Stunden bei Normaldruck und $50^{\circ} \mathrm{C}$. Danach werden die Membranen gründlich mit Wasser gewaschen und über Nacht bei $100^{\circ} \mathrm{C}$ zur Aktivierung getrocknet.

Als Abstandshalter zwischen Enzym und aktivierter Silikat-Oberfläche wird der Spacer Glutardialdehyd (2,5 Ma.\%) verwendet. Die Anbindung des Dialdehyds erfolgt bei RT und einem Druck von 600 mbar für eine Stunde. Während dieser Reaktion kann eine gleichmäßige rot-violette Verfärbung der Membran beobachtet werden.

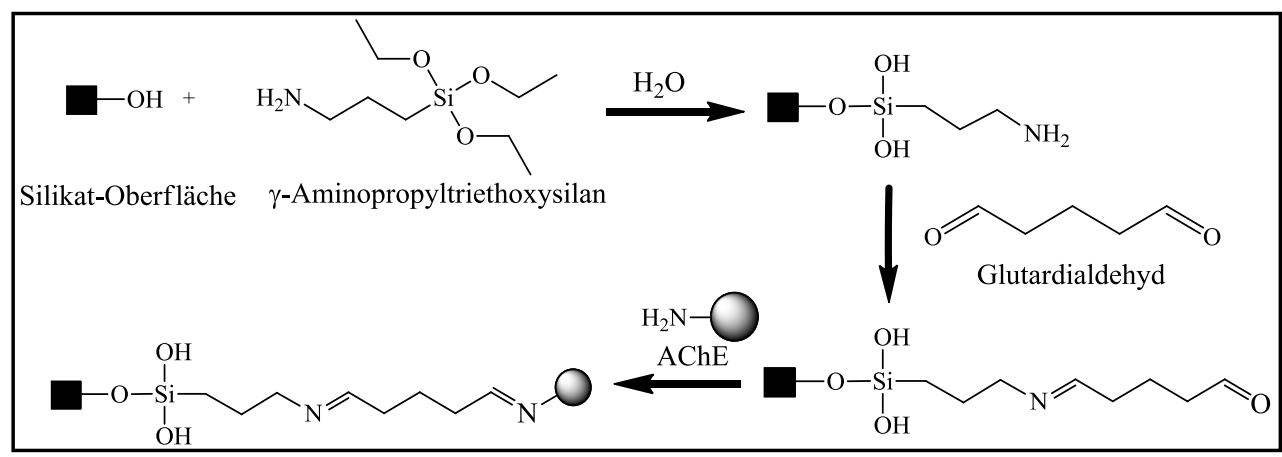

Bild 2 Schema der Enzym-Immobilisierungsreaktion 
Die Enzym-Immobilisierung wird bei $0^{\circ} \mathrm{C}$ und $600 \mathrm{mbar}$ in einer Phosphatpufferlösung mit einem $\mathrm{pH}-$ Wert von 7 durchgeführt. Die Funktionalisierungs-parameter sind für beide Matrix-Materialien identisch.

Die Aktivität der Enzym-Silikat-Komplexe wird mit einer Acetylcholinchlorid-Lösung $(0,2 \mathrm{M})$, die mit einer Dinatriumphosphatlösung $(0,01 \mathrm{M})$ auf $\mathrm{pH} 7$ eingestellt wird, bei $25^{\circ} \mathrm{C}$ innerhalb von 10 Minuten bestimmt. Die vermessenen Membranen werden anschließend für 10 Minuten in eine Carbofuranlösung getaucht, mit Phosphatpuffer gespült und unter denselben Bedingungen erneut vermessen.

\section{Ergebnisse}

\subsection{Texturcharakterisierung mittels Quecksilberintrusion}

Das Bild 3 zeigt die Graphen der Texturcharakterisierung des Ausgangsmaterials nach der DruckLösungsmittelaustauschsynthese (a) und des Ausgangsmaterials nach der Hydrothermalbehandlung (b) mittels Quecksilber-Intrusion. Es ist deutlich zu sehen, dass die mittels Quecksilber-Intrusion nicht nachweisbaren Mikroporen zu Mesoporen aufgeweitet werden. Außerdem wird der Porendurchmesser der Makroporen nur gering beeinflusst. Die zugehörigen Texturdaten sind in Tafel 2 zusammengefasst.
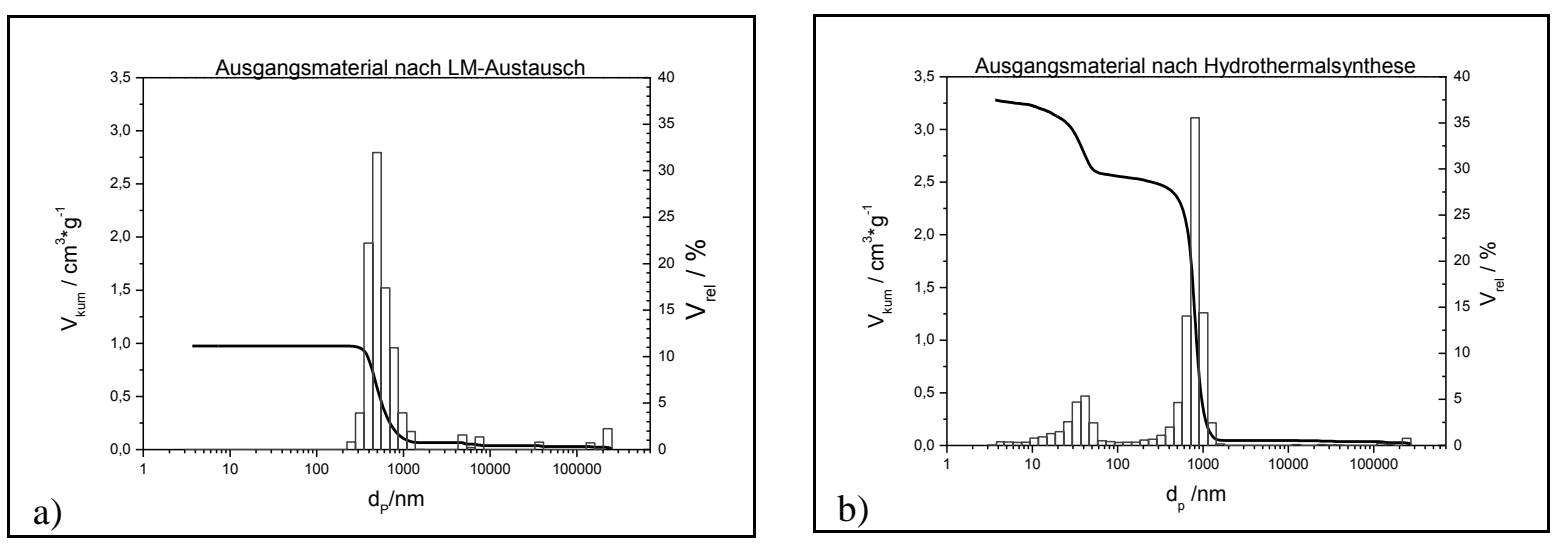

Bild 3 Ergebnisse zur Quecksilber-Intrusion

Tafel 2 Texturdaten bestimmt mittels Quecksilber-Intrusion

\begin{tabular}{l|c|c}
\hline & $\mathrm{d}_{\mathrm{P} \text { Meso }}[\mathrm{nm}]$ & $\mathrm{d}_{\mathrm{P} \text { Makro }}[\mathrm{nm}]$ \\
\hline Ausgangsmaterial nach Lösungsmittel-Austausch & - & 738 \\
\hline Ausgangsmaterial nach Hydrothermalsynthese & 45,8 & 853 \\
\hline
\end{tabular}

\subsection{Vergleich Enzym-CPG- und Enzym-Sol-Gel-Komplex mittels pH-Messung}

Die Oberflächenfunktionalisierung mit dem Enzym dient als Nachweis für Pestizide. Die Pflanzenschutzmittel inhibieren die Funktion des Enzyms und verändern dadurch deren Aktivität, die über eine enzymkatalysierte Substratzersetzung als Testreaktion verfolgbar ist. Im Beispiel Acetylcholinesterase entsteht bei der Substratzersetzung Essigsäure. Dies kann mittels pH-Messung verfolgt werden.

$$
\text { Acetylcholin }+\mathrm{H}_{2} \mathrm{O} \stackrel{\mathrm{AChE}}{\longrightarrow} \text { Essigsäure }+ \text { Cholin }
$$

Im Vergleich mit einer porösen Glasmembran (Bild 4a) erzielt das neue Material (Bild 4b) bezogen auf die spezifische Oberfläche und fast gleiche Reaktionsporengröße $(50 \mathrm{~nm})$ eine schnellere Ansprechzeit, was den Einfluss der Makroporen als Transportporen beweist. 

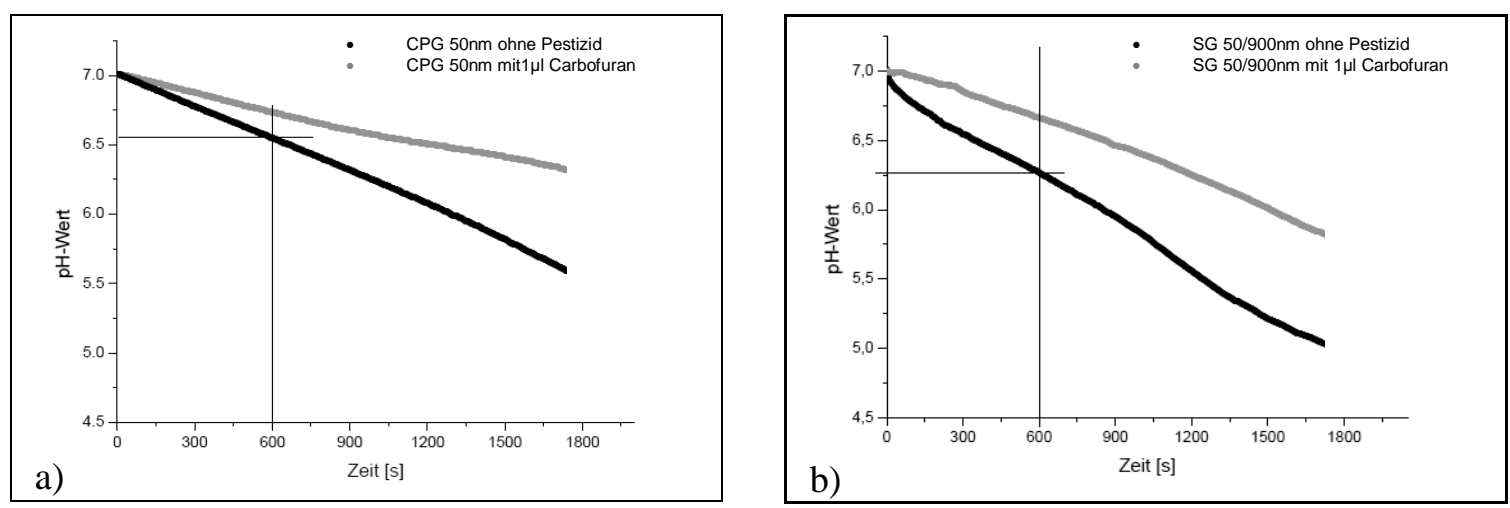

Bild 4 pH-Zeit-Diagramm für die enzymkatalysierte Zersetzung von Acetylcholin

Die Inhibierung des Enzym-Sol-Gel-Komplexes für das Testpestizid Carbofuran konnte mit einer Nachweisgrenze von $0.125 \mu \mathrm{g} / \mathrm{l}$ gezeigt werden. Für den Nachweis der Langzeitstabilität werden die immobilisierten Membranen bei $4^{\circ} \mathrm{C}$ in einer Pufferatmosphäre mit einem pH-Wert von 7 gelagert. Die Membranen zeigen nach 7 Wochen noch nahezu die Anfangsaktivität.

\section{Ausblick}

Da der Enzym-Membran-Komplex Teil der Wegwerf-Komponente eines Sensors wäre, ist es sinnvoll die Immobilisierung des Enzyms und die Enzymkonzentration weiter zu optimieren. So besteht die Möglichkeit, ein Enzym-Membran-Komplex für ein bestimmtes Pestizid oder eine festgelegte Pestizidkonzentration (gesetzl. Rückstandhöchstmenge) maßzuschneidern. Außerdem kann parallel zum Enzym ein Indikator, der die pH-Wertänderung anzeigt, auf der Membran Co-Immobilisiert werden.

\section{Literatur}

[1] N. Anders, Diplomarbeit, Martin-Luther-Universität Halle-Wittenberg, (2010)

[2] W. Fichtner, M. Berthold, R. Müller, H. Kaden, D. Enke, T. Hahn, Neue Herausforderungen und Anwendungen in der Sensortechnik, TUDpress, 279, (2005)

[3] D. Enke, K. Hobritz, F. Janowski, W. Fichtner, M. Berthold, H. Kaden, Dresdner Beiträge zur Sensorik, 29, (2007), 235-238

[4] H. Preising, D. Enke, Chemie Ingenieur Technik, 78/9, (2006), 1333 